\title{
BENEFIT OF THE INTEGRATION OF VISIBLE AND THERMAL INFRARED IMAGES FOR THE SURVEY AND ENERGY EFFICIENCY ANALYSIS IN THE CONSTRUCTION FIELD
}

\author{
Claudio Parente ${ }^{1 *}$, Massimiliano Pepe ${ }^{2}$ \\ ${ }^{1}$ University of Naples "Parthenope", Italy \\ ${ }^{2}$ Politecnico di Bari, Italy
}

Infrared thermography is a non-invasive and non-contact technique that provides surface temperature distribution by measuring infrared radiation emitted by a body. The instrument that can determine the surface temperature of the object indicated is the thermal-camera. Nowadays, the use of thermal imaging is increasingly widespread and its application fields are numerous, especially in the construction environment. In this way, using a modern thermal-camera, it has showed in this paper the high performance of temperature map generated by these instruments. The integration of thermal camera with optical sensors allows to obtain, by a suitable methodology, a high quality of thermal image. Indeed, taking in account large building, it is impossible to cover the whole building and at the same time acquire with a high geometric resolution in relation to the modern thermal camera. In order to identify anomalies temperature on large and modern structure, a case study of the use of thermal images building is showed in the manuscript. Indeed, the knowledge of temperature distribution on the facade of the building provides very useful information to discover many hidden conditions related to the building performance, maintenance and energy efficient. Because the construction field accounts for $40 \%$ of energy demand in Europe, the use of thermal images in this environment provides an important contribute in the analyses process of temperature archived on the buildings.

Key words: thermal images, tir, structure from motion, emissivity, pan-sharpening

\section{INTRODUCTION}

Remotely sensed Thermal InfraRed (TIR) data have been widely used in a wide range of applications, such as surveillance and reconnaissance, inspections of buildings and civil infrastructures, restoration and management activities of either cultural heritage monuments or buildings [1].

Thermal InfraRed sensors transform the thermal energy, emitted by an object in the infrared band of the electromagnetic spectrum, into a visible image [2]. Indeed, any object at an absolute temperature greater than zero emits infrared radiation. The emitted radiation is a function of the temperature of the material: the higher the temperature, the greater the intensity of the infrared energy emitted.

TIR sensors record the temperatures emitted by the objects in infrared region, which can be subdivided in the following spectral bands:

- Near-infrared (NIR) from $0.78 \mu \mathrm{m}$ to $1 \mu \mathrm{m}$;

- Short-wavelength infrared (SWIR) from $1 \mu \mathrm{m}$ to $3 \mu \mathrm{m}$;

- Mid-wavelength infrared (MWIR) from $3 \mu \mathrm{m}$ to $6 \mu \mathrm{m}$.

- Long-wavelength infrared (LWIR) from $6 \mu \mathrm{m}$ to $15 \mu \mathrm{m}$;

- Very Long-wavelength infrared (VLWIR) from $15 \mu \mathrm{m}$ to $1000 \mu \mathrm{m}$.

However, this classification is not standardized. Indeed, confusion in area of limits and further division of sub-ranges of optical radiation is particularly clear in case of infrared radiation range. More proposals on divi- sion of infrared range have been published in the literature. The division shown above is based on the limits of spectral bands of commonly used infrared detectors [3].

The thermal sensors available on the market acquire in the MWIR and LWIR region. The reasons are related to the atmospheric transmittance and the band of peak emissions. In the first case, the infrared radiation that travels through air has a low absorption by various air particles. In the second case, the wavelength at which electromagnetic radiation is emitted depends on the temperature of the object. In other words, the higher the temperature, the shorter its wavelength [4]. Considering the temperature achieved in the most common thermal applications, the infrared wavelength ranges between $7-15 \mu \mathrm{m}$. So, working in this range, the IR thermography can be considered a non-contact and non-intrusive technique of investigation. Considering the dimensions of the thermal sensors frame, the area covered by each image is small. In this way, in order to increase the geometric resolution, more solutions have been proposed. Many works present in literature propose the integration of different sensors. Indeed, some authors [5, 6]suggest the integration of 3D laser scanner, a thermal camera and a color camera to obtain thermal maps. Other authors $[7,8]$ using Structure-from-Motion (SFM) approach and the data fusion techniques between thermal and color images were able to build 3D thermal model. Based on the fusion of thermal and color images obtained by different sensors, in this paper a novel and easy approach is explained. In particular, the paper is organized as fol- 
lows. Section called "Physical background" illustrates the basic concepts of thermal infrared energy. Section "Data and method" explains, by a case study, the process that is necessary to develop in order to obtain high quality of thermal orthophoto. Conclusions are summarized at the end of the paper.

\section{PHYSICAL BACKGROUND}

\section{Thermal infrared energy}

Temperature is the measure of the degree of heat or cooling of a body [9]. The temperature is related, at the atomic/molecular level, to internal body activity (activity of the particles that make up the object). The energy of particles of matter in random motion is called kinetic heat (also referred to as internal real or true heat). All objects having a temperature above absolute zero $(0 \mathrm{~K}$; $-273.16{ }^{\circ} \mathrm{C}$; $-459.69{ }^{\circ} \mathrm{F}$ ) exhibit this random motion. It is possible to measure the true kinetic temperature (Tkin) or concentration of this heat using a thermometer [10].

When the molecules collide, they change their state energy and the energy is released in the form of electromagnetic radiation, which constitutes the so-called radiant flux. Ultimately, the radiant temperature (Trad) can be considered the measurement of the radiant flow out of a body. A black body is a theoretical model where radiant temperature and kinetic temperature coincide:

$T_{\text {rad }}=T_{\text {kin }}$

Indeed, the black body is an idealized physical body that absorbs all the incident radiation (regardless of the wavelength considered). The relationship between the wavelength of spectral exitance and temperature for a black body is given by Wien's displacement law:

$\lambda(m)=\frac{A}{T}$

Where $\lambda(m)$ is the wavelength of maximum spectral radiant, A constant $(2898 \mu \mathrm{mK})$ and $\mathrm{T}$ the temperature.

The spectral distribution of the energy radiated from the surface of a black body at various temperature is described by Stefan-Boltzmann law:

$M=\int_{0}^{\infty} M(\lambda) d \lambda=\sigma T^{4}$

Where $M$ is total radiance exitance, $M(\lambda)$ spectral radiance exitance, $\sigma$ Stefan-Boltzmann constant (5.6697 x 10-8 $\mathrm{Wm} 2 \mathrm{~K}-4$ ) and $\mathrm{T}$ temperature of black body (expresses in $\mathrm{K})$.

However, all the real materials do not behave as blackbodies because they can emit only a fraction of the energy emitted from a black body at equivalent temperature. The ratio from the radiant exitance of a material (at a given temperature) and the radiant exitance of black body (at some temperature) is called emissivity (e). Also, every real material shows a specific emissivity value. In this way, it is possible to obtain a new equation of radiance exitance M [11]:
$M=e \sigma T_{\text {kin }}{ }^{4}=\sigma T_{\text {rad }}{ }^{4}$

This defines the relation between the radiant temperature and kinetic temperature of a body as:

$T_{\text {rad }}=e^{1 / 4} T_{k i n}$

Taking in account the emissivity of the material, the specific spectral emission $\mathrm{M}(\lambda)$ of an object can be obtained by Planck's emission law:

$$
M(\lambda)=e_{\lambda} \frac{c_{1}}{\lambda^{5}} \frac{1}{\exp \left(\frac{c_{2}}{\lambda T}\right)-1}
$$

Where $c_{1}$ and $c_{2}$ are emission constant $\left(c_{1}=3.7418 \cdot 104\right.$ $\left.\mathrm{W} \cdot \mathrm{cm} 2 \cdot \mu \mathrm{m} 4, \mathrm{c}_{2}=1.4388 \cdot 104 \mathrm{~K} \cdot \mu \mathrm{m}\right), \mathrm{T}$ is the absolute temperature (expressed in $\mathrm{K}$ ) and $\lambda$ the wavelength (expresses in $\mu \mathrm{m})$.

Inverting Planck's equation, it is possible to obtain the temperature value as shown below:

$$
T=\frac{c_{2}}{\lambda \ln \left(\left[e_{\lambda} c_{1} \lambda^{-5} / M(\lambda)\right]+1\right)}
$$

\section{Emissivity}

Emissivity is a relationship between two similar energies. Therefore, it is a dimensional magnitude where the values can (theoretically) range from 0 to 1 . A value of emissivity equal to 1 means that the material can absorb all of the incident energy and then emit it as if the object was a black body. Emissivity equal to zero implies that the body does not absorb any radiation within the electromagnetic spectrum: this would be the case of an ideal reflector (or even called ideal white body).

The emissivity of an object may be influenced by a number factors, such as the color (darker colored objects have a higher emissivity than lighter colored objects), surface roughness and moisture content [12]. Indeed, when the spectral component of the radiant energy interacts with a semi-transparent surface, the radiation may be reflected, absorbed and/or transmitted. The reflectivity $(\rho)$ is a property that determines the fraction of the incident radiation that is reflected by the surface, the absortivity $(\alpha)$ is the fraction of the irradiation absorbed by the surface and the transmissivity $(\mathrm{T})$ is the fraction of the irradiation that is transmitted through the surface [13]. Because these parameters depend on the wavelength, it is possible obtain the following equation:

$\alpha_{\lambda}+\rho_{\lambda}+\tau_{\lambda}=1$

In according to the conservation of energy and Kirchhoff's law, the emissivity of a surface is equal its absorptivity [14]. This mean that $a_{\lambda}=\varepsilon_{\lambda}$ and, of consequence, the previous equation becomes:

$\varepsilon_{\lambda}+\rho_{\lambda}+\tau_{\lambda}=1$ 
Generally, for opaque bodies the transmissivity is zero. So, reconsidering the equation (9) in relation to the emissivity, it is possible to obtain the following important relation:

$\varepsilon_{\lambda}=1-\rho_{\lambda}$

The emissivity values of some common materials used in building environment, are shown in the table 1.

Table 1: Coefficient of emissivity of some building materials (Fluke manual [14])

\begin{tabular}{|c|c|}
\hline Material & Emissivity \\
\hline Aluminum polished & 0.05 \\
\hline Aluminum strongly oxidized & 0.25 \\
\hline Brass dull tarnished & 0.22 \\
\hline Brick, common & 0.85 \\
\hline Bronze porous rough & 0.55 \\
\hline Bronze polished & 0.10 \\
\hline Concrete & 0.54 \\
\hline Copper polished & 0.01 \\
\hline Electrical tape black plastic & 0.95 \\
\hline Formica & 0.93 \\
\hline Glass & 0.92 \\
\hline Glass frosted & 0.96 \\
\hline Gold polished & 0.02 \\
\hline Steel rough surface & 0.96 \\
\hline
\end{tabular}

\section{DATA AND METHOD}

\section{Study area}

The case study concerns the principal façade of the University on Naples "Parthenope" located in the "Centro Direzionale" of Naples city (Italy). The building was built in the late 90's and is characterized by a unique body. This structure has a regular (quadrangular) shape and is divided in seven levels with internal vertical connections. The principal façade (north façade) that is the object of the survey, is represented by symmetrical and simple rectangular geometric lines, where the various levels of the building are easily distinguished. The only element that interrupts the vertical pattern is represented by central glass windows, which also identifies and highlights the entrance to the building. On the ground floor of the entire façade, there is the portico which is constituted by circular monolithic columns. This part encloses the first two levels of the building. The ground floor is identified by pieces of marble and porthole windows, while the first level is distinguished by continuous glass windows. The upper levels are characterized by panels and windows.

\section{Thermal camera sensor}

Nowadays there are on the market of increasingly high-performance thermal cameras at an attractive price. For this experimentation, an IR Thermo-Camera Fluke Tir1 was used for thermal analyses. The Fluke TiR1 thermal imager provides high quality images and stable temperature readings. Indeed, the Fluke TiR1 thermal imager measures temperatures ranging from -20 to $100^{\circ} \mathrm{C}$ and it has a thermal sensitivity of less than or equal to $0.07^{\circ} \mathrm{C}$ at $30^{\circ} \mathrm{C}$. This elevate sensitivity allows the users to see even small temperature differences and, as consequence, could indicate any problems. The Fluke TiR1 thermal imager has a 3.6" landscape color VGA (Video Graphics Array) screen with a resolution of $640 \times 480$ pixels. The value of Istantenuos Field Of View (IFOV) of the TiR1 sensor is $2.5 \mathrm{mrad}$ [15]. This latter parameter allows to determinate the acquisition distance between the building and the sensor. Indeed, the optical system of an infrared sensor collects the infrared energy from a circular measurement spot and focuses it on the detector. Also, the Ground Sample Distance (GSD) can be defined by the product of the distance from the sensor to the object and the size of the spot being measured (d). Let the IFOV value of the TiR1 sensor, it is possible to build the table 1 where are reported the values of geometric resolution varying the distance object-sensor, using the following relation [16]:

$$
\mathrm{GSD}=d \cdot I F O V
$$

Table 2: Distance object-sensor and ground sample distance

\begin{tabular}{|c|c|}
\hline $\begin{array}{c}\text { Distance object-sensor } \\
(\mathrm{m})\end{array}$ & $\begin{array}{c}\text { Ground Sample } \\
\text { Distance } \\
(\mathrm{m})\end{array}$ \\
\hline 1 & 0.003 \\
\hline 2 & 0.005 \\
\hline 3 & 0.008 \\
\hline 5 & 0.013 \\
\hline 8 & 0.020 \\
\hline 10 & 0.025 \\
\hline 20 & 0.050 \\
\hline 30 & 0.075 \\
\hline 50 & 0.125 \\
\hline
\end{tabular}

Therefore, as shown in the table 2, increasing the distance decreases the geometric resolution of the thermal image. However, in the thermal context it is important to emphasize that the emissivity is related to the distance from the sensor to the object [17]. This means that if the resolution decreases, the pixel temperatures of the same area will tend toward the mean temperature derived from averaging the pixel temperatures from higher resolution images. 


\section{Method}

In order to obtain accuracy and high geometric resolution thermal data of the structure under investigation, more aspects, geomatics and technologies were considered. In particular, the procedure developed in order to achieve this goal, can be summarized in the workflow reported in the Figure 1.

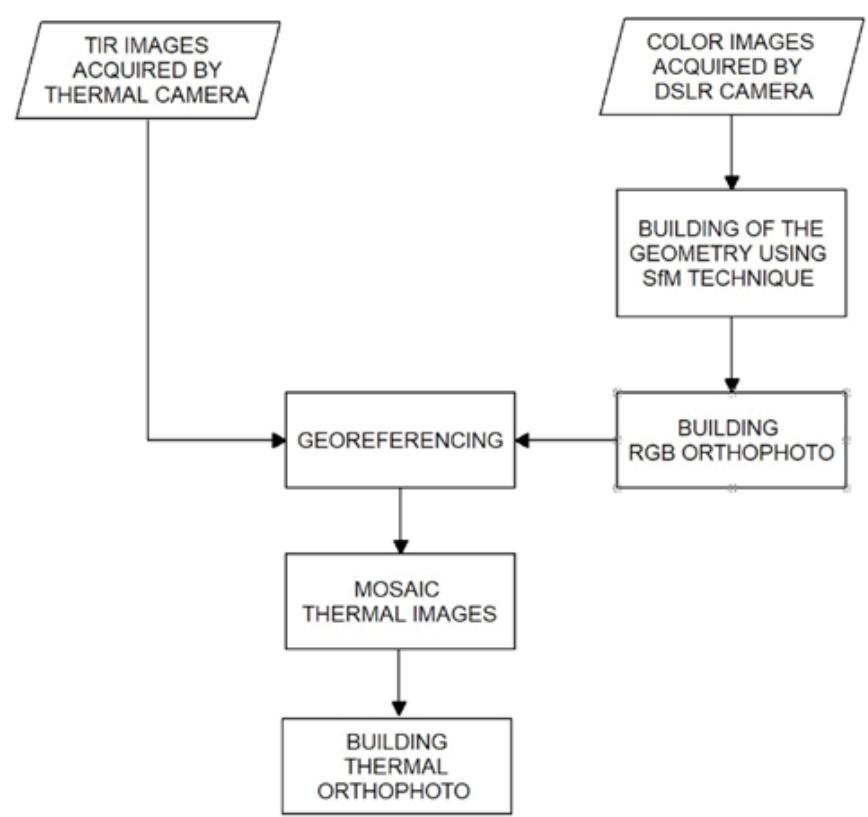

Figure 1: Workflow of the methodology implemented

As shown in the previous workflow, it was necessary to integrate the images obtained by thermal and visible sensors. Also, the first step was to determine the geometric characteristics of the structure (building) taken into consideration. This task was obtained by Close Range Photogrammetry (CRP) technique[18, 19]. In this environment, the so called "Structure-from-Motion" (SfM) approach has become quite popular in close-range photogrammetry because it allows to build three-dimensional models in a semiautomatic way from a set of images. Based on computer vision algorithms, the SfM approach requires a block of images with a high degree of overlap that capture the object of the survey from several locations [20]. An important task in the SfM process consists in the recovery of the calibration parameters of the camera which the metric structure of the scene cannot be calculated. The traditional pattern-based calibration procedure is laborious and often impractical. A more flexible and elegant approach, known as camera "self-calibration", consists in the recovery of the unknowns' parameters solely using point correspondences across images [21]. Actually, a variety of software applications can automatically perform camera self-calibration and, at the same time, to produce dense point clouds and/or 3D models, suitable for various photogrammetric applications. Using SFM technique, it was possible to perform the survey of the façade investigated. In particular, the photogrammetry survey was carried out by Pentax K-x
DSLR camera with $18-55 \mathrm{~mm}$ f/3.5- 5.6 lens and a total amount of 115 images were captured. Using total station Leica TCRP 1201+ (angle measurement accuracy equal to 1 " while the distance measurement accuracy without reflector is $2 \mathrm{~mm}+2 \mathrm{ppm}$ ) and Leica GeoOffice software, it was possible to calculate (in a local coordinate system) the coordinates of some points easily recognizable on images (markers). The photogrammetric processing was performed with AgisoftPhotoScan software where the 3D model reconstruction was obtained by several straightforward processing steps. Indeed, the first task realized in this environment was the alignment of the acquired images (Figure 2).

In this phase, due the important reflectance of some part of the building and/or the presence of obstacles between the building and the camera, it was necessary to realize many masks on the single images. Aligned the images, 6 marker points were used as Ground Control Points in order to realize a metric model. The average Root Mean Square (RMS) achieved on the marker was $0.010 \mathrm{~m}$. Subsequently, the dense point cloud, using "Very High" setting, was realized. Once obtained the point clouds, a surface mesh was created. Also, after the geometry (i.e. the mesh) was constructed, the 3D model of the building was textured and used for orthophoto generation of the building façade.

The colour orthophoto or RGB (Red-Green-Blue) orthophoto of the building façade, obtained by SfM approach, is shown in the Figure 3.
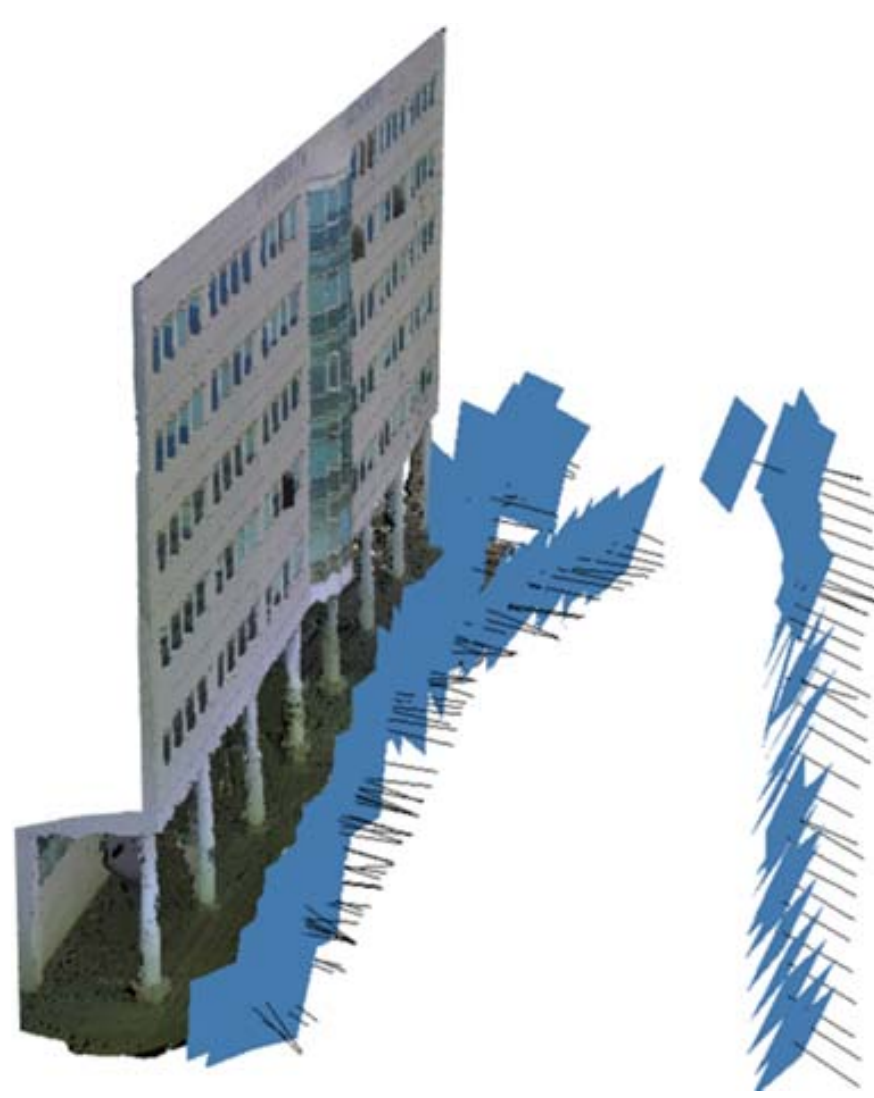

Figure 2: Position of the cameras on 3D textured model 


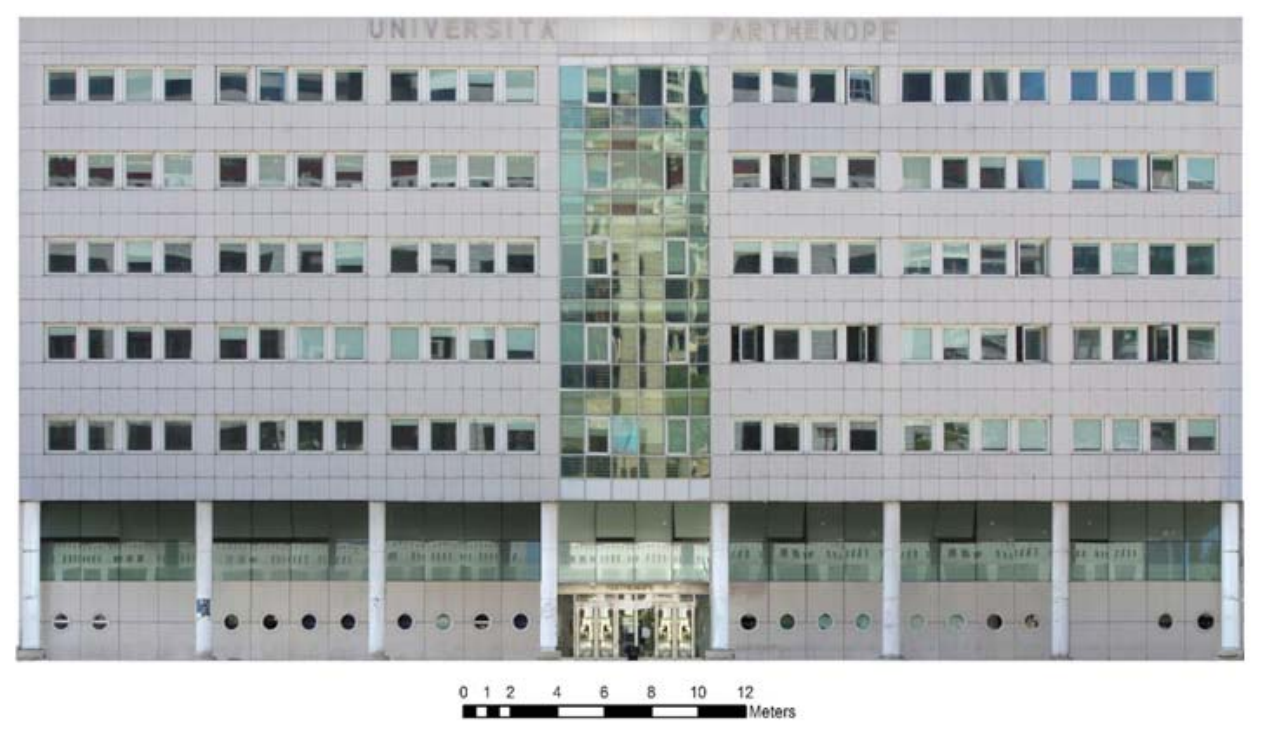

Figure 3: Colour orthophoto of the building façade

The thermal survey was carried out the 17 th July 2017 around the $3.00 \mathrm{pm}$ (local time). The thermal dataset consists of 12 images. The thermal images were processed through the use of specific software called SmartView (version 4.1) that allowed to obtain the temperatures of the surfaces. In order to make the visible image of the object acquired by TIR, the camera makes a conversion in false colours. In general, there are several types of visualization that can be selected inside the sensor or computer software during image analysis. Palette called "iron" are the standard to display the images. In this representation, the colour scale starts from black to indicate the coldest spots or parts, as the temperature increases the colour becomes blue, red, orange, yellow and finally white for the hottest spots. In addition, the SmartView software was allowed to report on the thermal images the markers which represent the minimum (blue colour), maximum (red colour) and average temperature (white colour) of the surface investigated. Figure 4 shows a typical example of thermal images of the area study. In order to obtain a 3D model, the same approach used for optical sensor images was applied to thermal images. The resulting model was unsatisfactory. Indeed, Agisoft software was not able to align the images due the poor

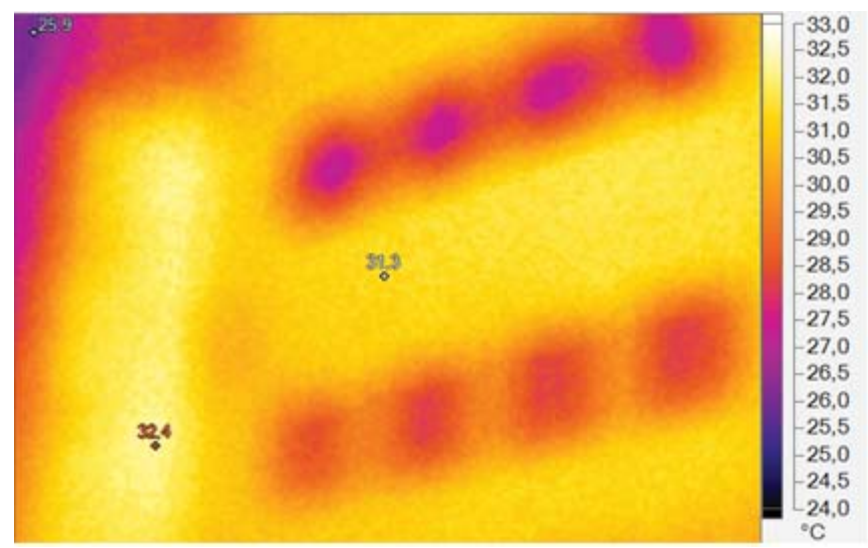

Figure 4: Thermal image of the building in SmartView environment correlation between the thermal images, despite the elevate overlap during the stage of the acquisition. In addition, in the SfM approach, the correlation between the colour images increase if the angle of convergence is great [22]. Because the emissivity is related to the angle of acquisition of the sensors [23], it follows that this approach should be inaccurate for the measure of the temperature.

Also, to overcome this situation, the thermal images were georeferenced on the colour orthophoto in ArcMap environment using the projective transformation. The projective transformation introduces two plane coordinate systems using a central projection. All projection rays are straight lines through the perspective centre and the equations that allow this type of transformation are:

$$
\begin{gathered}
x=\frac{a_{1} x^{\prime}+a_{2} y^{\prime}+a_{3}}{c_{1} x^{\prime}+c_{2} y^{\prime}+1} \\
y=\frac{b_{1} x^{\prime}+b_{2} y^{\prime}+b_{3}}{c_{1} x^{\prime}+c_{2} y^{\prime}+1}
\end{gathered}
$$

Where $x, y$ are the coordinates of thermal original images, $x^{\prime}, y^{\prime}$ are coordinates of rectificated thermal images in a local frame and $a_{1}, a_{2}, a_{3}, b_{1}, b_{2}, b_{3}, c_{1}, c_{2}$ are the projective parameters.

Because the unknown in the equations (11) are 8, it follows that in the projective transformation, a minimum of 4 control points (or 4 links) are request and no three of which may lie on a common straight line. In order to obtain a higher quality of the transformed image, a minimum of 8 points were taken in consideration. In this case the system of equations was solved by least squares adjustment. From the statistical point of view, the average RMS error value achieved on all thermal images was 0.080 pixel and the standard deviation RMS was 0.018 pixel. The single orthophoto was generated using a pixel size of $5 \mathrm{~cm}$. Subsequently, all the thermal images were merged in order to obtain a unique image. The result of 


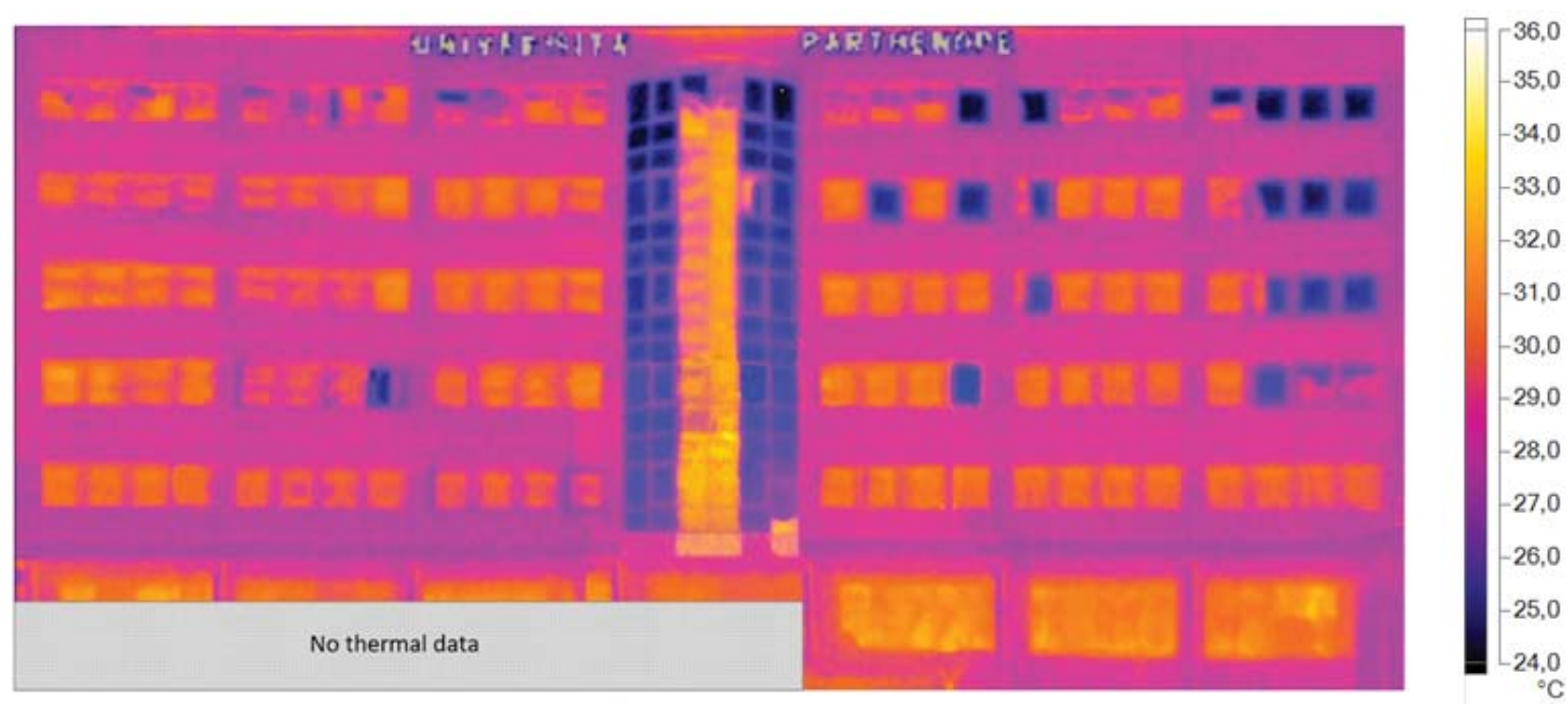

Figure 5: Thermal orthophoto of the building façade investigated

this operation using a false colour image (Iron shades), is shown in the Figure 5.

Unfortunately, as it is possible to note in the Figure 5, it was not possible to acquire thermal images in a portion of the structure. This is due to presence of the garden in front of the building contained a vegetation quite height that prevented the acquisition by the thermal sensor from a suitable distance. In addition, it is necessary to consider even technological limits related to the small dimension and long focal length of the thermal sensor used for the experimentation.

So, analyzing the thermal orthophoto (Figure 5), it is possible to note how some windows (blue color) showed a lower temperature than other (orange color). This is due to the activity of air conditioners which produce air cool. In this context, it is possible to identify, from a point of view of energy efficiency, the rooms that showed an energy dispersion. In addition, it is easy to identify the difference of the temperature due to divers material of which the structure is made: the glass windows (belong the room where the air condition in cool mode has been not used and indicated in orange color in the Figure 5) had a greater temperature than panels.

\section{Pan-sharpening approach}

In order to increase the resolution of the thermal images, some authors $[24,25]$ suggest the use of pan-sharpening method. Indeed, the pan-sharpening allows to fuse the higher geometric resolution of the panchromatic image with the spectral resolution of multispectral (thermal) image. Also, in order to increase the geometric resolution of the thermal orthophoto, pan-sharpening task was performed in Geographic Information System (GIS) environment by IHS (Intensity-Hue-Saturation) method [26, 27] and using a suitable weights dataset [28].

The intensity component I is substituted by the panchromatic image, which was obtained by optical sensor. The new component Red, Green and Blue of the thermal colour image (R'T, G'T, B'T) with the same geometric resolution of panchromatic data was produced using the following equation:

$$
\left[\begin{array}{c}
R_{T}^{\prime} \\
G_{T}^{\prime} \\
B_{T}^{\prime}
\end{array}\right]=\left[\begin{array}{c}
R_{T}^{\prime}+\delta \\
G_{T}^{\prime}+\delta \\
B_{T}^{\prime}+\delta
\end{array}\right]
$$

Where

$\delta=P A N-I$

and

$I=\frac{R_{T}^{\prime}+G_{T}^{\prime}+B_{T}^{\prime}}{3}$

Therefore, the above relationships were implemented in ArcMap software using raster calculator tool. The result of the thermal pan-sharpening is shown in the Figure 6a. A visual comparison of a particular between the simple thermal orthophoto and the thermal orthophoto obtained through the pan-sharpening, is shown in Figures $6 \mathrm{~b}$ and $6 \mathrm{c}$. However, if on the one hand this operation is correct from the informatics point of view, from the other side it is not correct from the physical point of view. Indeed, this approach changes the values of the colour and, as consequence, the temperature values. In addition, this approach is not correct because the panchromatic band cover only a visible part of the spectral wavelength range. Indeed, the pan-sharpening technique is widespread applied in the study and observation of the images generated by satellite sensors, such as Worldview, Ikonos, Sentinel2, Landsat etc. in order to increase the geometric resolution of the multispectral bands in the range of the electromagnetic spectrum covered by panchromatic band. Therefore, the only advantage of pan-sharpening operation in this environment is to better identify object shape in the area under investigation. Indeed, as just es- 


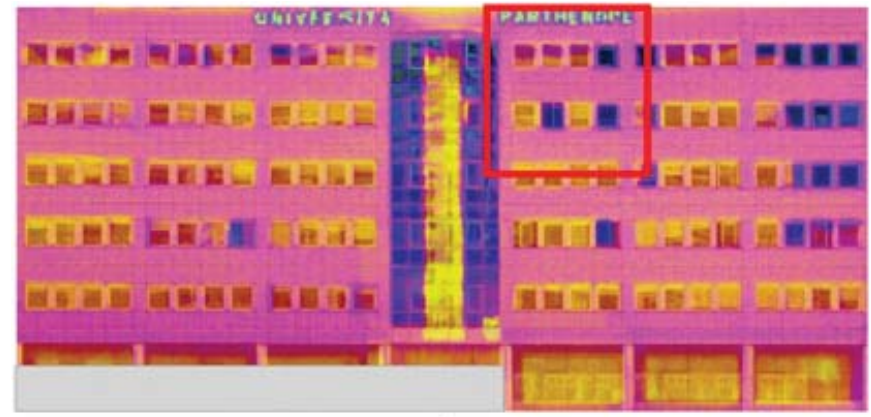

a)

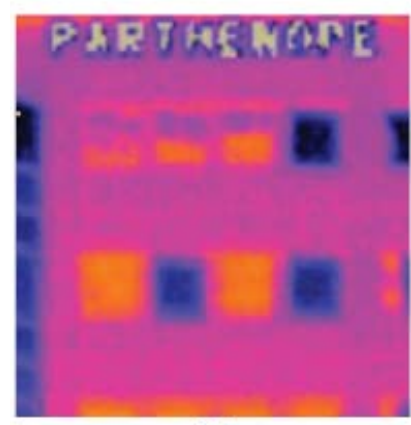

b)

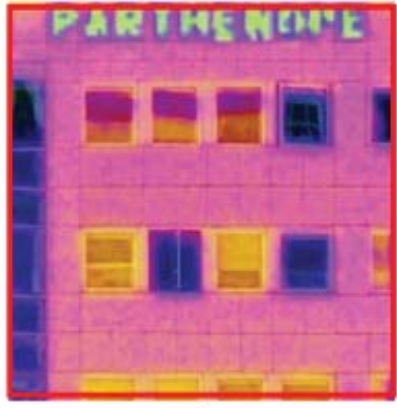

c)
Figure 6:

a) Pan-sharpening applied on the entire building façade; b) particular of the thermal orthophoto;

c) particular of the pan-sharpening thermal orthophoto

tablished, it is impossible to make measure of temperature on pan-sharpening image.

\section{CONCLUSIONS}

The methodology developed has allowed to obtain thermal images with an elevated radiometric and geometric resolution. This operation has been possible thanks to the integration of photogrammetric and thermal surveytechniques. As shown in the case study, the use of suitable procedure has provided very useful information in the analysis of the temperature of the investigated structure. In addition, considering the low cost of the hardware components and the knowledge in this field, it is desirable to increasingly use of temperature monitoring in order to improve the energy efficiency of buildings. Indeed, sensitivity to energy issues related to the construction sector is becoming more and more important.

Also, the thermal survey of the investigated building allowed to identify clearly the differences between the several windows belonging to the structure. In this way, it was possible to monitor the improper use of energy sources.

Lastly, considering the criticalities related to pan-sharpening operation for thermal applications, it is desirable to manage these several data (termal and oprical) in GIS environment that allows to integrate as well as to distinguish different information levels.

\section{REFERENCE}

1. Lerma, J. L., Mileto, C., Vegas, F., \& Cabrelles, M. (2007, October). Visible and thermal IR documentation of a masonry brickwork building. In XXI International CIPA Symposium (pp. 01-06).

2. Zissis, G. J., \& Wolfe, W. L. (1978). The infrared handbook. Infrared Information And Analysis Center Ann Arbor Mi.

3. Chrzanowski, K. (2010). Testing Thermal Imagers Practical Guidebook.

4. Usamentiaga, R., Venegas, P., Guerediaga, J., Vega, L., Molleda, J., \& Bulnes, F. G. (2014). Infrared thermography for temperature measurement and non-destructive testing. Sensors, 14(7), 1230512348. https://doi.org/10.3390/s140712305

5. Borrmann, D., Afzal, H., Elseberg, J., \& Nüchter, A. (2012). Mutual calibration for 3D thermal mapping. IFAC Proceedings Volumes, 45(22), 605-610.

6. Oreifej, O. J., Cramer, A., Zakhor A. (2014). Automatic generation of $3 d$ thermal maps of building interiors. ASHRAE transactions, 120, C1.

7. Scaioni, M., Rosina, E., L'Erario, A., \& Dìaz-Vilariño, L. (2017). INTEGRATION OF INFRARED THERMOGRAPHY AND PHOTOGRAMMETRIC SURVEYING OF BUILT LANDSCAPE. International Archives of the Photogrammetry, Remote Sensing \& Spatial Information Sciences, 42. https://doi. org/10.5194/isprs-archives-XLII-5-W1-153-2017

8. Maset, E., Fusiello, A., Crosilla, F., Toldo, R., \& Zorzetto, D. (2017). Photogrammetric 3d Building Reconstruction from Thermal Images. ISPRS Annals of the Photogrammetry, Remote Sensing and Spatial Information Sciences, 4, 25.. https://10.5194/ isprs-annals-IV-2-W3-25-2017

9. Quattrochi, D. A., \& Luvall, J. C. (2009). Thermal remote sensing in Earth science research (pp. 64-78). SAGE Publications Ltd.: London, UK.

10. Jensen, J. R. (2007). Remote sensing of the environment: an earth resource perspective: Pearson Prentice Hall. Upper Saddle River, NJ.

11. Prakash, A. (2000). Thermal remote sensing: concepts, issues and applications. International Archives of Photogrammetry and Remote Sensing, 33(B1; PART 1), 239-243.

12. Reddy, M. A., \& Reddy, A. (2008). Textbook of remote sensing and geographical information systems (p. 453). Hyderabad: BS publications.

13. Bergman, T. L., Incropera, F. P., DeWitt, D. P., \& Lavine, A. S. (2011). Fundamentals of heat and mass transfer. John Wiley \& Sons. 
14. Fluke, Fluke Building Diagnostic Thermal Imagers, Datasheet. Available on line: http://www.myflukestore.com/pdfs/cache/www.myflukestore.com/fluke/ thermal_imager/tir1_9hz/datasheet/fluke_tir1_9hz_ thermal_imager_datasheet.pdf, 2017 (last accessed 19 November 2017).

15. Barreira, E., de Freitas, V. P., Delgado, J. M. P. Q., \& Ramos, N. M. M. (2012). Thermography applications in the study of buildings hygrothermal behaviour. In Infrared Thermography. InTech. https:// doi.org/10.5772/28282

16. Koretsky, G. M., Nicoll, J. F., \& Taylor, M. S. (2013). A tutorial on electro-optical/infrared (EO/IR) theory and systems (No. IDA-D-4642). INSTITUTE FOR DEFENSE ANALYSES ALEXANDRIA VA.

17. Ball, M., \& Pinkerton, H. (2006). Factors affecting the accuracy of thermal imaging cameras in volcanology. Journal of Geophysical Research: Solid Earth, 111(B11).https://doi.org/10.1029/2005JB003829

18. Luhmann, T., Robson, S., Kyle, S., \& Boehm, J. (2013). Close-range photogrammetry and 3D imaging. Walter de Gruyter.

19. Pepe, M., \& Parente, C. (2017). Cultural Heritage Documentation in Sis Environment: an application for" Porta Sirena" in the archaeological site of Paestum. International Archives of the Photogrammetry, Remote Sensing \& Spatial Information Sciences, 42. https://doi.org/10.5194/isprs-archivesXLII-5-W1-427-2017.

20. Pepe, M., Fregonese, L., \& Scaioni, M. (2018). Planning airborne photogrammetry and remote-sensing missions with modern platforms and sensors. European Journal of Remote Sensing, 51(1), 412-435. https://doi.org/10.1080/22797254.2018.1444945

21. AI-ISMAEIL, K. (2011). Structure from Motion \& Camera Self-Calibration (Doctoral dissertation, Université de Bourgogne).
22. Fraser, C. (2015). Advances in Close-Range Photogrammetry, Photogrammetric Week'15.

23. Nunak, T., Rakrueangdet, K., Nunak, N., \& Suesut, T. (2015, March). Thermal image resolution on angular emissivity measurements using infrared thermography. In Proceedings of the International MultiConference of Engineers and Computer Scientists (Vol. 1, pp. 18-20).

24. Lagüela, S., Armesto, J., Arias, P., \& Herráez, J. (2012). Automation of thermographic 3D modelling through image fusion and image matching techniques. Automation in Construction, 27, 24-31. https://doi.org/10.1016/j.autcon.2012.05.011

25. Luhmann, T., Piechel, J., \& Roelfs, T. (2013). Geometric calibration of thermographic cameras. In Thermal Infrared Remote Sensing (pp. 27-42). Springer, Dordrecht. https://doi.org/10.1007/978-94007-6639-6_2

26. Rahmani, S., Strait, M., Merkurjev, D., Moeller, M., \& Wittman, T. (2010). An adaptive IHS pan-sharpening method. IEEE Geoscience and Remote Sensing Letters, 7(4), 746-750. https://doi.org/10.1109/ LGRS.2010.2046715

27. Pepe, M., \& Parente, C. [2018]. BURNED AREA RECOGNITION BY CHANGE DETECTION ANALYSIS USING IMAGES DERIVED FROM SENTINEL-2 SATELLITE: THE CASE STUDIO OF SORRENTO PENISOLA, ITALY. Journal of Applied Engineering Science, 16(2), 225-232.

28. Parente, C., \& Pepe, M. (2017). Influence of the weights in IHS and Brovey methods for pan-sharpening WorldView-3 satellite images. International Journal of Engineering \& Technology, 6(3), 71-77. https://doi.org/10.14419/ijet.v6i3.7702 